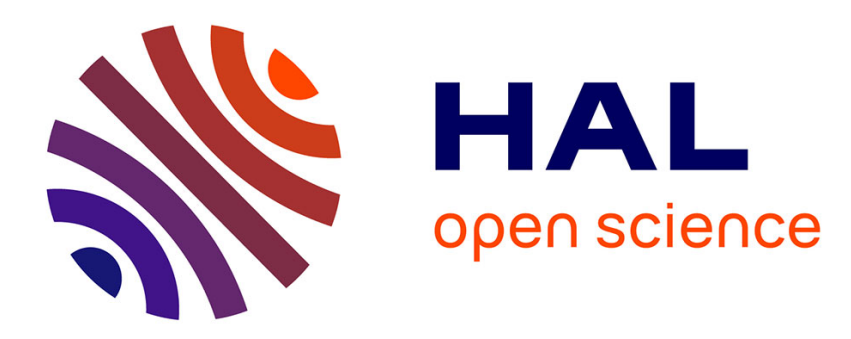

\title{
Time-frequency localization from sparsity constraints
}

Pierre Borgnat, Patrick Flandrin

\section{To cite this version:}

Pierre Borgnat, Patrick Flandrin. Time-frequency localization from sparsity constraints. 2007. ensl00176412

\section{HAL Id: ensl-00176412 https://hal-ens-lyon.archives-ouvertes.fr/ensl-00176412}

Preprint submitted on 3 Oct 2007

HAL is a multi-disciplinary open access archive for the deposit and dissemination of scientific research documents, whether they are published or not. The documents may come from teaching and research institutions in France or abroad, or from public or private research centers.
L'archive ouverte pluridisciplinaire HAL, est destinée au dépôt et à la diffusion de documents scientifiques de niveau recherche, publiés ou non, émanant des établissements d'enseignement et de recherche français ou étrangers, des laboratoires publics ou privés. 


\title{
TIME-FREQUENCY LOCALIZATION FROM SPARSITY CONSTRAINTS
}

\author{
Pierre Borgnat \& Patrick Flandrin \\ École Normale Supérieure de Lyon, Laboratoire de Physique (UMR 5672 CNRS) \\ 46 allée d'Italie 69364 Lyon Cedex 07 France \\ \{pborgnat, flandrin\}eens-lyon.fr
}

\begin{abstract}
In the case of multicomponent AM-FM signals, the idealized representation which consists of weighted trajectories on the time-frequency (TF) plane, is intrinsically sparse. Recent advances in optimal recovery from sparsity constraints thus suggest to revisit the issue of TF localization by exploiting sparsity, as adapted to the specific context of (quadratic) TF distributions. Based on classical results in TF analysis, it is argued that the relevant information is mostly concentrated in a restricted subset of Fourier coefficients of the Wigner-Ville distribution neighbouring the origin of the ambiguity plane. Using this incomplete information as the primary constraint, the desired distribution follows as the minimum $l_{1}$-norm solution in the transformed TF domain. Possibilities and limitations of the approach are demonstrated via controlled numerical experiments, its performance is assessed in various configurations and the results are compared with standard techniques. It is shown that improved representations can be obtained, though at a computational cost which is significantly increased.
\end{abstract}

Index Terms - time-frequency, localization, sparsity

\section{TIME-FREQUENCY LOCALIZATION}

\subsection{AM-FM signals as time-frequency trajectories}

If we consider a signal made of the superimposition of a finite number of AM-FM components:

$$
x(t)=\sum_{k=1}^{K} a_{k}(t) e^{i \varphi_{k}(t)}
$$

it is natural to attach to it an idealized TF distribution (TFD) $\rho(t, f)$ which essentially distributes the total energy along TF trajectories according to:

$$
\rho(t, f)=\sum_{k=1}^{K} a_{k}^{2}(t) \delta\left(f-\dot{\varphi_{k}}(t) / 2 \pi\right) .
$$

In such a picture, each component is characterized at each time instant by essentially one instantaneous frequency (which, in a first approximation, can be identified to the phase derivative), weighted by the corresponding instantaneous power.

Except for very special cases, there is no general methodology to automatically get a distribution as in (1). In the case of a single component $(K=1)$, it is well-known [6] that a perfect localization can be attained for pure FM signals with a linear modulation $\left(a_{1}(t)=1\right.$ and $\left.\dot{\varphi}_{1}(t)=f_{0}+\alpha t\right)$ by using the Wigner-Ville Distribution (WVD):

$$
W_{x}(t, f)=\int_{-\infty}^{+\infty} x\left(t+\frac{\tau}{2}\right) x^{*}\left(t-\frac{\tau}{2}\right) e^{-i 2 \pi f \tau} d \tau .
$$

Although this property can be extended to some forms of nonlinear FMs (e.g., Bertrands' distributions for power-laws [6]), it is generally at the expense of a substantially increased complexity in the definition (and the computation) of the distributions, with furthermore the limitation of being adapted to some specific type of FM only and to not extend to multicomponent situations. For this last point, the well-known drawback of energy distributions is to obey a quadratic superposition principle which creates cross-terms in between any two components of a signal, and thus significantly reduces the readability of Wigner-type distributions $[6,7]$.

\subsection{Classical techniques of TF localization}

The aforementioned difficulties have led to many developments during the last 20 years but, unfortunately, since both localization and creation of cross-terms result from the very same mechanism [7], it turns out that trying to impose simultaneously localization and cross-terms reduction is faced with a trade-off that can be viewed as a form of time-frequency uncertainty principle. The simplest way to understand where this trade-off comes from and how to manage it is to interpret the WVD in its 2D Fourier transform plane.

By definition, the WVD admits a 2D Fourier transform which is referred to as the ambiguity function (AF) and reads

$$
A_{x}(\xi, \tau)=\int_{-\infty}^{+\infty} x\left(t+\frac{\tau}{2}\right) x^{*}\left(t-\frac{\tau}{2}\right) e^{i 2 \pi \xi t} d t .
$$

If we introduce the TF shift operator $\mathbf{T}_{\xi, \tau}$ which acts on signals $x(t) \in L^{2}(\mathbb{R})$ as

$$
\left(\mathbf{T}_{\xi, \tau} x\right)(t):=x(t-\tau) e^{-i 2 \pi \xi(t-\tau / 2)},
$$


we readily get that $A_{x}(\xi, \tau)=\left\langle x, \mathbf{T}_{\xi, \tau} x\right\rangle$ and, by construction, the AF can thus be viewed as a TF correlation function. As such, an AF exhibits most properties of a correlation function, including hermitian symmetry and the fact that its modulus is maximum at the origin. Moreover, in the case of multicomponent signals, the total AF consists of both auto-components neighbouring the origin of the plane and cross-components mostly located at a TF distance from the origin which directly depends on the TF separation between the individual components and that are the Fourier images of the undesired cross-terms in the TF plane. This observation early prompted [5] to propose improvements upon the WVD by weighting the AF around the origin of the plane prior applying an inverse 2D Fourier transform: the more restricted the weighting domain, the more effective the crossterms suppression but, at the same time, the more decreased the TF localization. Although this procedure (which defines Cohen's class [6] on geometrical grounds) proved reasonably effective, other approaches have also been proposed, which basically exploit the phase information usually discarded in simple quadratic distributions such as spectrograms (or scalograms). In this respect, reassigned spectrograms [8] proved in particular extremely efficient to approach (1) and will thus be used in Section 3 for a sake of comparisons.

\subsection{A sparsity perspective}

If the analyzed signal is given in discrete-time and supposed to be of dimension $N$ in time, its TFD is of dimension $N^{2}$ when computed over $N$ frequency bins. However, assuming that $K \ll N$, i.e., that the number of components is much smaller than the dimension of the signal, the targeted TFD which is supposed to satisfy (1) is distributed over the plane in a very sparse way, with only $K 1 \mathrm{D}$ trajectories where at most $K . N$ values are expected to be non-zero. Imposing such a sparsity is therefore a new way of approaching the problem.

\section{LOCALIZATION FROM SPARSITY CONSTRAINTS}

\subsection{Principle}

The principle of the approach is very simple. It consists in selecting a suitable collection of AF samples neighbouring the origin of the plane in a given domain $\Omega(\xi, \tau)$ and searching for the sparsest TFD $\rho$ such that its 2D Fourier transform $\mathcal{F}\{\rho\}$ coincides with the original AF over $\Omega$. Looking for a perfectly spiky solution such as (1) would require to minimize the total number of non-zero coefficients, i.e., the $l_{0}$-norm of the TFD. While this turns out not to be practicable from a computational viewpoint, a series of recent works [3, 4] have shown that a near-optimal solution can be attained at a sensibly more affordable cost by minimizing the $l_{1}$-norm, reducing the problem to the solving of a linear program. It is this technique which is proposed to be followed here, the desired localized TFD $\rho(t, f)$ being therefore the solution of the constrained minimization problem:

$$
\min _{\rho}\|\rho\|_{1} ; \mathcal{F}\{\rho\}-A_{x}=\left.0\right|_{(\xi, \tau) \in \Omega}
$$

At this point, it is worth emphasizing that the specific context we are interested in makes the proposed approach slightly different from a classical recovery problem from an incomplete Fourier description. Indeed, the AF is by definition the 2D Fourier transform of the WVD, and there is no point in recovering the latter from the knowledge of the AF over $\Omega$. The rationale is rather that $\Omega$ conveys the essential information about local features of the constitutive components of the signal and discards cross-terms that limit readability. The resulting TFD $\rho(t, f)$ is therefore more "constructed" by the procedure than "reconstructed" since it defines an idealized object which does not exist per se prior optimization.

\subsection{Constraints}

Exact vs. approximate - The primary constraint which is given by (3) imposes a strict equality over $\Omega$ in the AF domain. This however can be relaxed [4] according to

$$
\min _{\rho}\|\rho\|_{1} ;\left\|\mathcal{F}\{\rho\}-A_{x}\right\|_{2} \leq\left.\epsilon\right|_{(\xi, \tau) \in \Omega},
$$

where $\epsilon$ is a user-specified bound. Both possibilities (3) and (4) will be considered in the following.

Selection of Fourier samples - As far as the specification of the domain $\Omega$ is concerned, a number of different possibilities are also offered, in terms of both area and shape. Based on Theorem 1.3 of [3], the cardinality of $\Omega$ should be

$$
\operatorname{card}(\Omega)=O\left(K \cdot N \cdot \log \left(N^{2}\right)\right)
$$

for the recovery of $K$ AM-FM trajectories of $N$ points each in a TF domain of size $N^{2}$. In practice, results given in [3] suggest that the logarithmic term can be replaced by a constant in between 4 and 8 . Stressing again the fact that a perfect recovery of the WVD is not our objective and that as much AF values outside from the origin as possible are to be discarded, this constant term should be preferably chosen smaller. As justified in the forthcoming Section 3, it proved reasonably efficient in the simulations we conducted to choose $\operatorname{card}(\Omega) \approx N$.

Concerning the shape of the AF domain onto which the $1 / 0$ mask with $O(N)$ non-zero elements is to be applied to the AF, we chose here the simplest solution which is to make use of a fixed square geometry. A refined procedure would consist in selecting a domain whose geometry matches the (data-dependent) structure of the AF near the origin (e.g., with a Radially Gaussian Kernel [1]), but this will not be followed up here because of space limitation.

Additional constraints - One particular interest of the approach based on optimization is that further constraints can 

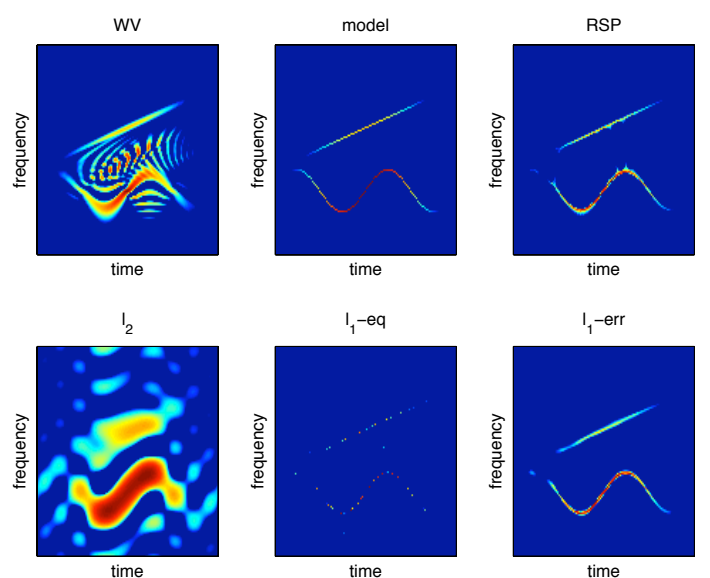

Fig. 1. Synthetic example - Different TFDs are displayed in the case of a 128 points signal whose TF model (1) is given in the middle of the top row, in between the Wigner-Ville Distribution (left) and a reassigned spectrogram (right). The bottom row presents the results obtained from optimizations based on the only knowledge of the $13 \times 13$ Fourier samples of the WVD neighbouring the origin of the AF plane. From left to right: minimum $l_{2}$-norm, exact minimum $l_{1}$-norm according to (3) and approximate minimum $l_{1}$-norm according to (4) with $\epsilon=0.05\|x\|_{2}$. For all diagrams, amplitudes are color coded logarithmically, with a dynamic range of $18 \mathrm{~dB}$.

be imposed besides (3) or (4). One can think, e.g., of the marginalization properties attached to unit cross-sections in the AF plane [6]. This however is not necessarily relevant in the context of a sharp TF localization since, in the case of multicomponent signals, highly oscillatory behaviours along TF trajectories will be favoured. A more interesting variation is to favor regular time evolutions, what can be achieved explicitly by imposing specific AF cross-sections or, implicitly, by using as a starting point a time smoothed WVD.

\section{EXAMPLES}

The feasability of the method has been tested on simple, yet informative examples. All the computations have been made in MATLAB, with the TIME-FREQUENCY TOOLBOX ${ }^{1}$ for the TF computations and the $l_{1}$-MAGIC TOOLBOX ${ }^{2}$ for the optimization.

Figure 1 compares different TFDs in the case of a $N=$ 128 points signal made of the superimposition of a linear and of a sinusoidal FM, both modulated in amplitude with a Gaussian. In this example, the different optimizations have been based on the only knowledge of the $13 \times 13$ Fourier samples

\footnotetext{
${ }^{1}$ http: //tftb. nongnu.org

${ }^{2}$ http: //www.11-magic.org
}
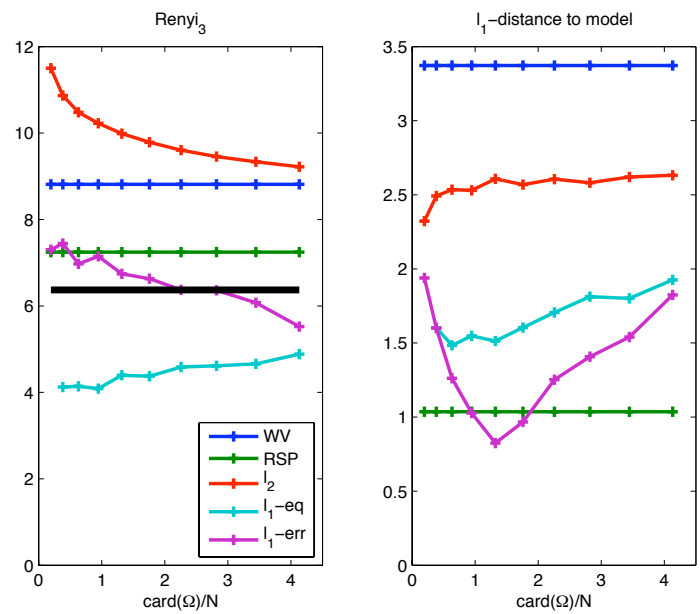

Fig. 2. Rényi entropy and $l_{1}$-distance - Using the same signal as in Fig. 1, the localization properties and proximity from the model of the different TFDs are quantified in terms of the Rényi entropy of order 3 (left, with the model entropy in thick black line) and $l_{1}$-distance (right), as a function of the relative number of AF samples card $(\Omega) / N$ used in the optimizations.

of the WVD neighbouring the origin of the AF plane, i.e., on a subset of about $1 \%$ only of the total number of AF coefficients. From a qualitative point of view, it turns out from this Figure that the approximate minimum $l_{1}$-norm solution (according to (4)) is very effective, even as compared to the reassigned spectogram which is known to usually behave best for this kind of signal (and whose window length has been optimized so as to best fit the model in the $l_{1}$-norm sense). This appreciation can be quantified further in terms of both the achieved performance with respect to the actual model, and the influence of the cardinality of the AF domain from which the optimization is conducted. This is reported in Figure 2 which displays both a localization measure (the Rényi entropy of order 3 [2]) and the $l_{1}$-distance to the model as a function of $\operatorname{card}(\Omega) / N$. What is revealed by this Figure is that both minimum $l_{1}$-norm solutions are generally better localized (i.e., have a smaller Rényi entropy) than the other considered TFDs, with even an entropy that might be smaller than the model one whose value is in this case 6.37. In particular, the exact $l_{1}$ solution has always the minimum entropy but, as evidenced by Fig. 1, this is due to an oversparse, discontinuous, structure which results in a larger distance to the actual model. The evolution of this distance shows that the best behavior is obtained with the approximate minimum $l_{1}$-norm solution, the minimum being obtained for $\operatorname{card}(\Omega) \approx N$. Additionnally, it is worth noticing that, while the WVD we started with is known to attain negative values and whereas no positivity constraint has been imposed, the minimum $l_{1}$ norm solutions happen to be almost positive. 

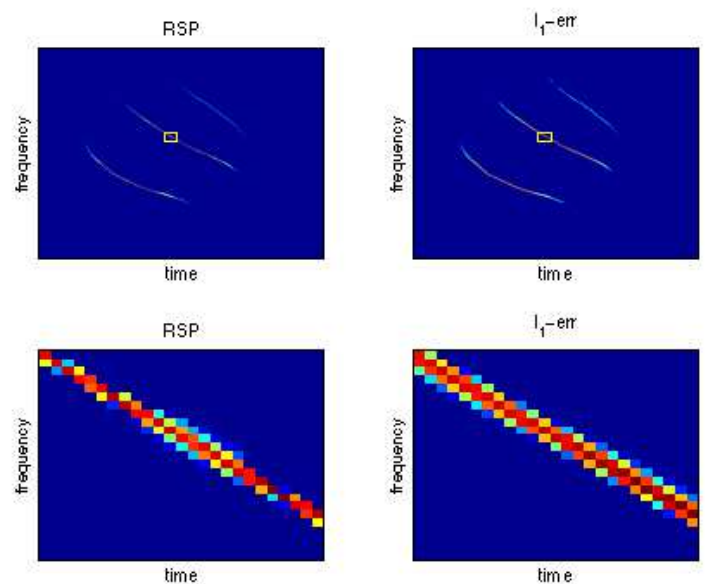

Fig. 3. Real data example - This Figure compares a reassigned spectrogram (left column) and the approximate minimum $l_{1}$-norm solution (4) with $\epsilon=0.05\|x\|_{2}$ (right column) in the case of a bat echolocation call of effective length $N=400$, the optimization being based on the knowledge of the $23 \times 23$ Fourier samples of the WVD neighbouring the origin of the AF plane. The bottom row displays enlarged versions of the distributions within the yellow boxes in the top row. For all diagrams, amplitudes are color coded logarithmically, with a dynamic range of $18 \mathrm{~dB}$.

As a complement to the synthetic example of Fig. 1, Figure 3 is concerned with some real data, namely the classical benchmark signal of a bat echolocation call ${ }^{3}$ of effective length $N=400$. In this case too, the approximate minimum $l_{1}$-norm solution (4) with $\operatorname{card}(\Omega)=23 \times 23 \approx 1.3 \mathrm{~N}$ compares very favorably with a reassigned spectrogram in terms of localization, with even some smoother regularity along TF trajectories.

Given the above reported findings, the new discussed approach is no doubt attractive in terms of ability to give sharply localized TFDs in the case of AM-FM multicomponent signals. There is however a price to pay for this performance, which is a quite heavy computational cost. For illustrating this point, Table 1 reports the average computation times associated to the simulations of Fig. 2: one can see that, under similar conditions, the $l_{1}$-based TFDs differ from classical ones by several orders of magnitude.

\section{CONCLUSION AND PERSPECTIVES}

A new approach has been proposed for getting sharply localized TFDs in the case of multicomponent AM-FM signals by

\footnotetext{
${ }^{3}$ The authors wish to thank Curtis Condon, Ken White, and Al Feng of the Beckman Institute of the University of Illinois for the bat data and for permission to use it in this paper.
}

\begin{tabular}{ccccc}
\hline & WV & RSP & $l_{1}$-eq & $l_{1}$-err \\
\hline average time (sec.) & 0.16 & 0.30 & 52 & 175 \\
\hline
\end{tabular}

Table 1. Computational cost - This Table reports the average computation times associated to the simulations of Fig. 2. All computations have been performed with MATLAB R2007a in similar conditions (MacIntel Core2Duo 2.16 GHz).

making profit of the assumed sparsity of their energy distribution in the TF plane. Due to space limitations, only the principle of the method has been outlined and there is clearly plenty of room for more thorough investigations and further developments. The selection of the Fourier samples in the AF domain needs special attention in terms of both area and shape, and it is expected that the use of adapted kernels (as proposed, e.g., in [1]) might prove useful. Whereas the heavy computational load can be considered as a severe drawback, it is worth stressing again that one advantage of the optimization-based approach is that additional constraints can be envisioned so as to satisfy specific properties in the TF plane (such as, e.g., regularity conditions related to smoothed marginals). Those different points are under current investigation and will be reported elsewhere.

\section{REFERENCES}

[1] R.G. Baraniuk and D.L. Jones, "Signal-dependent timefrequency analysis using a radially Gaussian kernel," Signal Proc., Vol. 32, No. 3, pp. 263-284, 1993.

[2] R.G. Baraniuk, P. Flandrin, A.J.E.M. Janssen and O. Michel, "Measuring time-frequency information content using the Rényi entropies," IEEE Trans. on Info. Theory, Vol. 47, No. 4, pp. 1391-1409, 2001.

[3] E. Candès, J. Romberg and T. Tao, "Robust uncertainty principles: Exact signal reconstruction from highly incomplete frequency information," IEEE Trans. on Info. Theory, Vol. 52, No. 2, pp. 489-509, 2006.

[4] E. Candès and T. Tao, "Near-optimal signal recovery from random projections: Universal encoding strategies?," IEEE Trans. on Info. Theory, Vol. 52, No. 12, pp. 5406-5425, 2006.

[5] P. Flandrin, "Some features of time-frequency representations of multicomponent signals," IEEE Int. Conf. on Acoust., Speech and Signal Proc. ICASSP-84, San Diego (CA), pp. 41B.4.1-41B.4.4, 1984.

[6] P. Flandrin, Time-Frequency/Time-Scale Analysis. San Diego: Academic Press, 1999.

[7] P. Flandrin, "Cross-terms and localization in timefrequency energy distributions," in: Time-Frequency Signal Analysis and Processing (B. Boashash, ed.), Article 4.2, pp. 94-101, Elsevier, 2003.

[8] P. Flandrin, F. Auger and E. Chassande-Mottin, "TimeFrequency Reassignment - From Principles to Algorithms," in Applications in Time-Frequency Signal Processing (A. Papandreou-Suppappola, ed.), Chap. 5, pp. 179-203, CRC Press, 2003. 